\title{
Introduction of Sensor Spectral Response Into Image Fusion Methods. Application to Wavelet-Based Methods
}

\author{
Xavier Otazu, María González-Audícana, Octavi Fors, and Jorge Núñez
}

\begin{abstract}
Usual image fusion methods inject features from a high spatial resolution panchromatic sensor into every low spatial resolution multispectral band trying to preserve spectral signatures and improve spatial resolution to that of the panchromatic sensor. The objective is to obtain the image that would be observed by a sensor with the same spectral response (i.e., spectral sensitivity and quantum efficiency) as the multispectral sensors and the spatial resolution of the panchromatic sensor. But in these methods, features from electromagnetic spectrum regions not covered by multispectral sensors are injected into them, and physical spectral responses of the sensors are not considered during this process. This produces some undesirable effects, such as resolution overinjection images and slightly modified spectral signatures in some features. The authors present a technique which takes into account the physical electromagnetic spectrum responses of sensors during the fusion process, which produces images closer to the image obtained by the ideal sensor than those obtained by usual wavelet-based image fusion methods. This technique is used to define a new wavelet-based image fusion method.
\end{abstract}

Index Terms-Data fusion, image sensors, multispectral image, spectral response, wavelet transforms.

\section{INTRODUCTION}

$\mathbf{T}$ HE existence of a tradeoff between spatial and spectral resolution in remote sensing spaceborne imagery is well known. That is due to a combination of a set of observational constraints imposed by the acquisition system, detector specifications and satellite motion, among others. As a result, spaceborne imagery is usually offered to the community as two separate products: a high-resolution panchromatic (HRP) image and a low-resolution multispectral (LRM) image. In addition, an increasing number of applications, such as feature detection, change monitoring and land cover classification, often demand the highest spatial and spectral resolution for the best accomplishment of their objectives. In response to those needs, image fusion has become a powerful solution providing a single image

Manuscript received June 30, 2004; revised March 14, 2005. This work was supported in part by the programe Ramon y Cajal, founded by Spanish Science Ministery.

X. Otazu is with the Centre de Visió per Computador, Universitat Autònoma de Barcelona, 08193 Barcelona, Spain (e-mail: xotazu@cvc.uab.es).

M. González-Audícana is with Department of Projects and Rural Engineering, Escuela Técnica Superior de Ingenieros Agrónomos, Public University of Navarre, 31006 Pamplona, Spain (e-mail: maria.audicana@unavarra.es).

O. Fors and J. Núñez are with Departament d'Astronomia i Meteorologia, Universitat de Barcelona, E-08028 Barcelona Spain, and also with Observatori Fabra, E-08035 Barcelona, Spain (e-mail: ofors@am.ub.es; jorge@ am.ub.es). Digital Object Identifier 10.1109/TGRS.2005.856106 with simultaneously the multispectral content of the original LRM image and an enhanced spatial resolution.

Literature shows a large collection of fusion methods developed over the last two decades. Initial efforts based on component substitution (intensity-hue-saturation (IHS or LHS) [1]-[3], principal component substitution (PCS) [4]-[6]), or relative spectral contribution (intensity modulation (IM) [7], $\mathrm{PX}+\mathrm{S}$ on SPOT handbook [8], Brovey [9], [10]), are mainly focused on enhancing spatial resolution for easing tasks of human photointerpretation. However, it is not possible to undertake quantitative analysis of fused images obtained by those methods in a systematic way and with high degree of reliability, since the original multispectral content of the LRM image is greatly distorted.

Another family of methods is developed later trying to overcome this limitation. These operate on the basis of the injection of high-frequency components from the HRP image into the LRM image. This series is early initiated by the high-pass filtering (HPF) method [4], which provides far less spectral distortion with respect to its predecessors [11]. However, it is not until a second more recent stage that, with the upcoming of methods based on multiresolution analysis, fused products accomplish state-of-the-art results which can be employed for quantitative studies of their multispectral content (land-cover mapping [12] and urban areas mapping [13]-[22]). In Section II, we outline the different fusion methods whose decomposition algorithms are inscribed inside this category, and briefly discuss their distinctive nature.

However, no explicit physical information of the detection system has been taken into account in former fusion methods. In Section III, we present a technique which does incorporate information from the spectral response of the sensor in each band of the LRM image. This a priori knowledge is employed in the transformation model which injects spatial detail into the LRM image. This technique is used to define a new wavelet-based image fusion method. The proposed method retains other advantages of its predecessors, as its applicability to an arbitrary number of bands and its good behavior preserving spectral signatures of the LRM image.

In Section IV, the new method is applied to merge a QuickBird image of Sevilla, Spain. Its successful performance is shown through extensive quantitative assessment and complementary comparison with other methods. Conclusions are drawn in Section V. 


\section{Multiresolution-BASED AlgORITHMS}

The basic idea of all fusion methods based on wavelets is to extract the spatial detail information from the HRPimage not present in the LRM to inject it into the latter, usually using a multiresolution framework [23]-[25]. In the next subsection, we explain one of these methods, the additive wavelet LHS (AWL) method.

Different multiscale wavelet-based image fusion methods can be distinguished concerning the algorithm used to perform the detail extraction of the HRP image: based on decimated wavelet transform algorithms, used in [26]-[30] amongst others, and based on undecimated or redundant wavelet transform algorithms, used in [31]-[34].

The main difference between the decimated and undecimated fusion algorithms is the presence or absence of subsampling when the wavelet decomposition is performed. This subsampling causes a loss of linear continuity in spatial features such as edges, and the appearance of artifacts in those structures with neither horizontal nor vertical directions [33].

As shown in previous works [31]-[33], the undecimated algorithms are more appropriate for image fusion purposes than the decimated ones.

Besides, different wavelet-based image fusion methods can be distinguished, depending on the algorithm used to inject the spatial detail of the HRP image into the LRM one, as follows:

—injection directly into each LRM band, [26]-[31];

-injection into each LRM band through an IHS transform, [33]-[36];

-injection into each LRM band through a principal component analysis [32], [33], [36].

When the first methods are applied, the spatial detail of the HRP image is injected into each LRM band, so that the spatial information introduced into the whole LRM image is $n$ times that of the HRM image, $n$ being the number of spectral bands of the LRM sensor. Consequently, redundant spatial detail incorporation may appear when using these methods [32].

When methods based on IHS and PCA are used, the quality of the resulting merged images depends on the bandwidth of the HRP sensor. The best performance for IHS-based methods occurs when it covers the entire range of bandwidths of all the LRM bands (i.e., Ikonos and QuickBird panchromatic sensor) [33]. The use of a very narrow HRP band, as that of the SPOT $4 \mathrm{M}$ mode, is more favorable to PCA than to IHS [32].

In order to preserve, as much as possible, the spectral information of the multispectral image, different transformation models could be applied to the spatial detail information extracted from the HRP image before its injection into the LRM one. The simplest one is the identity model proposed in [37]. Recently, more complex models have been proposed in [31] and [38].

The combination of different wavelet decomposition algorithms, different spatial detail transformation models and different spatial detail injection procedures results in many different image fusion methods.

In fact, what all these image fusion methods aim for is to preserve as much as possible the spectral signature of objects, but the sensor spectral response has never previously been taken into account in this context. Here, we introduce a method which does incorporate such information in the fusion process, as well as the spectral signature of the physical observed object. We enumerate its main characteristics as follows.

- The undecimated à trous multiresolution wavelet decomposition algorithm [39] is used to decompose the HRP image and extract the spatial detail.

- The detail injection model tries to preserve the original LRM spectral signature in every pixel.

- Information from sensor spectral response is used in order to preserve spectral signatures and to determine the amount of spatial detail from the HRP image to inject into each corresponding LRM band.

\section{A. AWL Method}

One of the existing multiresolution wavelet-based image fusion methods is the AWL method [34]. It was originally defined for a three-band red-green-blue (RGB) multispectral image. In this method, the spectral signature is preserved since the HRP detail is injected into the luminance L-band of the original LRM image. This method can be summarized as follows.

1. Decompose original RGB image into the LHS color space, obtaining new L-, H-, and S-bands.

2. Perform a histogram matching between the HRP band and the L-band, modifying the former to obtain a new HRP band.

3. Decompose $\mathrm{HRP}^{\prime}$ into $n_{\omega}$ wavelet planes, i.e., $\mathrm{HRP}^{\prime}=\sum_{k=1}^{n_{\omega}} \omega_{k}+c, c$ being the residual plane.

4. Add HRP $\omega_{k}$ wavelet planes to the original L-band to obtain the merged $L^{f}$ band, i.e., $L^{f}=L+\sum_{k=1}^{n \omega} \omega_{k}$.

5. Obtain the final merged RGB image from the $L^{f} H S$ bands.

It is equivalent to inject the HRP detail into every RGB LRM band proportionally to their original values. It maintains the relative values between LRM bands, i.e., it tries to preserve spectral signature. The number $n_{\omega}$ of wavelet planes depends on the ratio between the spatial resolution of the original LRM and HRP bands, i.e., $n_{\omega}=\log _{2}\left(r_{\mathrm{LRM}} / r_{\mathrm{HRP}}\right), r_{\mathrm{LRM}}$ and $r_{\mathrm{HRP}}$ being the LRM and HRP spatial resolution, respectively.

The original AWL method is defined only for three band images, but it is easily extensible to $n$ bands in the following way:

$$
n_{i}^{f}=\frac{n_{i}}{\sum_{\forall i} n_{i}} \cdot \omega_{k}
$$

where $n_{i} \equiv \mathrm{LRM}_{i}$ is the original $\mathrm{LRM}_{i}$ band, $\omega_{k}$ is the $k$ th wavelet plane of the HRP band, and $n_{i}^{f}$ is the detail to inject into the $\mathrm{LRM}_{i}$ band. To simplify the notation, we use $n_{i}^{f}$ instead of $n_{i, k}^{f}$ because the method is applied to every $k$ th wavelet plane. In (1), the HRP detail injected into every LRM band is proportional to the LRM band original radiance value, that is, it maintains its spectral signature in the same way as AWL does with RGB images. This generalized proportional method is called proportional AWL (AWLP).

\section{B. Spectral Response}

It is important to include sensor spectral response information in image fusion methods for the following reasons.

- In order to preserve physical meaning of merged spectral bands, the sensor spectral response for each band has to 
be taken into account. It is not physically acceptable to include, for example, information from far, or even thermic, infrared, or even from microwave or radio bands into visual bands if the spectral response functions of the corresponding sensors do not overlap.

- Image fusion methods try to recover the image obtained by an ideal virtual sensor with the same spectral sensitivity of the LRM sensor and the same spatial resolution of the HRP sensor. This implies that the LRM spectral response has to be taken into account during the fusion process.

\section{Proposed Image-Fusion Method: WiSpeR Method}

The probability a photon of a given frequency is detected by a sensor system mainly depends on two factors: on the one hand, the physical properties of the observed object that emits or reflects the electromagnetic radiation and, on the other hand, the spectral response that characterizes the sensor system. This defines how efficiently the radiation is recorded by the sensor as a function of photon frequency $\nu$, i.e., the photon wavelength $\lambda=c / \nu, c$ being the speed of light. Throughout the paper, we indistinctly use the frequency and wavelength terms to refer to this concept. Other factors, e.g., atmospheric dispersion, modify the abovementioned probability; however, for the sake of simplicity, these will not be considered in the proposed fusion method.

In the next four subsections we formalize these two factors and how they can be algorithmically introduced in the fusion method we propose.

\section{A. Sensor Spectral Response}

The spectral response function (SRF) of a sensor defines the probability a photon of a given frequency is detected by this sensor. In our situation, two general SRFs exist: the SRF of the HRP sensor, designed as $\phi(\nu)$; and the SRF of the $i$ th band of the LRM sensor $\left(\operatorname{LRM}_{i}\right)$, designed as $\varphi_{i}(\nu), n_{c}$ being the number of LRM bands, $i=1, \ldots, n_{c}$. For the sake of simplicity, we hereafter refer $\varphi_{i}(\nu)$ as the SRF of the LRM sensor, and will omit the term band.

Let the event $m_{i}$ be the detection of a photon by the LRM sensor. The probability of the event $m_{i}$ is

$$
P\left(m_{i}\right)=\int \varphi_{i}(\nu) d \nu
$$

Similarly, the probability a photon is detected by the HRP sensor can be defined by the probability of the event $p$

$$
P(p)=\int \phi(\nu) d \nu
$$

In geometrical terms, the probability of the events $m_{i}$ and $p$ can be understood as the area below their corresponding SRFs.

Given the event $p$, the probability of the event $m_{i}$ is

$$
P\left(m_{i} \mid p\right)=\frac{P\left(m_{i} \cap p\right)}{P(p)}
$$

being

$$
P\left(m_{i} \cap p\right)=\int \min \left(\varphi_{i}, \phi\right) d \nu
$$

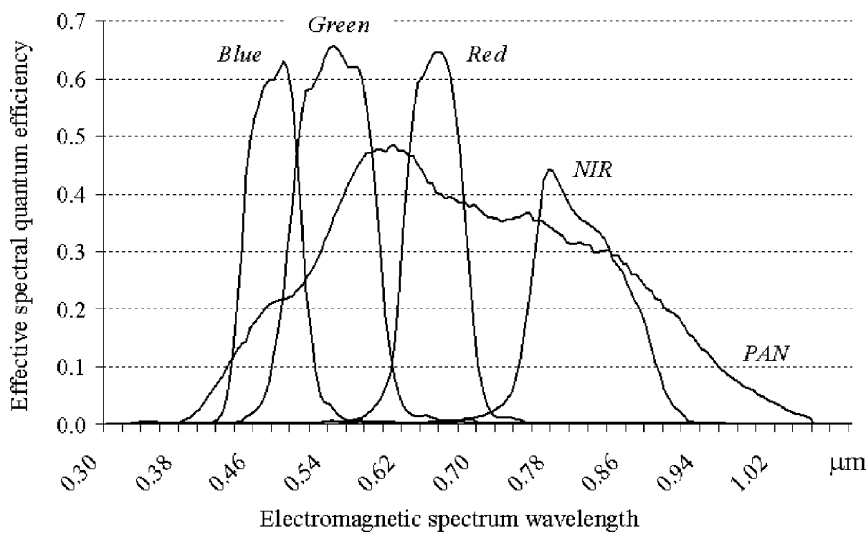

Fig. 1. SRFs of the QuickBird-2 multispectral (narrower functions) and panchromatic (wider function) sensors.

That is, given a photon detected by the HRP sensor (event $p$ ), the probability to be also detected by the LRM sensor (event $m_{i}$ ) is defined by the quotient between the intersection area of $\varphi_{i}(\nu)$ and $\phi(\nu)$ (see Fig. 1), and the area of the $\phi(\nu)$ function. Similarly, given a photon detected by the LRM sensor, the probability to be detected by the HRP sensor is

$$
P\left(p \mid m_{i}\right)=\frac{P\left(m_{i} \cap p\right)}{P\left(m_{i}\right)} .
$$

Note that (4) defines the fraction of HRP photons below the $\operatorname{LRM} \varphi_{i}(\nu)$ function. Similarly, (6) defines the fraction of $\mathrm{LRM}_{i}$ photons below the HRP $\phi(\nu)$ function.

Let $n_{i}$ be the number of photons detected by the LRM sensor. Let $n_{p, i}$ be the number of photons simultaneously detected by both the $\mathrm{LRM}_{i}$ sensor and the HRP sensor. The total number of photons detected by the HRP sensor should be

$$
n_{p}=\sum_{\forall i} n_{p, i}
$$

with

$$
n_{p, i}=P\left(p \mid m_{i}\right) \cdot n_{i}
$$

being the number of photons simultaneously below the $\phi(\nu)$ and the $\varphi_{i}(\nu)$ functions. From Fig. 1, (7) could be understood as $n_{p}$ being the sum of those $n_{i}$ photons simultaneously located below both the $\varphi_{i}(\nu)$ and the $\phi(\nu)$ functions, but this is not strictly correct.

Equation (7) only holds when $\bigcup_{i=1}^{n_{c}} P\left(m_{i} \cap p\right)=p$, i.e., when all the area below the $\phi(\nu)$ function is contained within the $\varphi_{i}(\nu)$ functions. Speaking in terms of photons, this expression is true when all the HRP-detected photons are going to be detected by some of the $\mathrm{LRM}_{i}$ sensors. This is not the general case because there are areas below the $\phi(\nu)$ function which are not below any of the $\varphi_{i}(\nu)$ functions.

If the number of HRP photons which are simultaneously below the $\phi(\nu)$ and $\varphi_{i}(\nu)$ functions is known, we can rewrite (4) and (6) in terms of these common photons as

$$
\begin{aligned}
& P\left(m_{i} \mid p_{m}\right)=\frac{P\left(m_{i} \cap p_{m}\right)}{P\left(p_{m}\right)} \\
& P\left(p_{m} \mid m_{i}\right)=\frac{P\left(m_{i} \cap p_{m}\right)}{P\left(m_{i}\right)}
\end{aligned}
$$


respectively, $p_{m}$ being the event corresponding to an HRP photon which is simultaneously below the $\phi(\nu)$ and the $\varphi_{i}(\nu)$ functions. In this case, since $m_{i} \cap p_{m}=m_{i} \cap p$, we have

$$
P\left(m_{i} \cap p_{m}\right)=P\left(m_{i} \cap p\right)=\int \min \left(\varphi_{i}, \phi\right) d \nu .
$$

The probability of event $p_{m}$ is

$$
P\left(p_{m}\right)=\int \min \left(\phi, \max \left(\varphi_{1}, \varphi_{2}, \ldots, \varphi_{n}\right)\right) d \nu .
$$

Let us define

$$
\alpha_{p}=\frac{\int \min \left(\phi, \max \left(\varphi_{1}, \varphi_{2}, \ldots, \varphi_{n}\right)\right) d \nu}{\int \phi(\nu) d \nu}
$$

as the fraction of the HRP photons, i.e., the photons below the $\phi(\nu)$ function, which are also below any of the $\varphi_{i}(\nu)$ functions. Given the number $n_{p}$ of HRP photons, the number of photons simultaneously detected by both this HRP sensor and the $\mathrm{LRM}_{i}$ sensor is

$$
n_{p, i}=P\left(m_{i} \mid p_{m}\right) \cdot \alpha_{p} n_{p} .
$$

This number of photons was also obtained in (8), but in that case the number $n_{i}$ of $\mathrm{LRM}_{i}$ photons were used. Rewriting (8) in terms of the new $p_{m}$ as

$$
n_{p, i}=P\left(p_{m} \mid m_{i}\right) \cdot n_{i}
$$

and combining it with (14), we finally obtain

$$
n_{i}^{\prime}=\frac{\alpha_{p} \cdot P\left(m_{i} \mid p_{m}\right)}{P\left(p_{m} \mid m_{i}\right)} \cdot n_{p} .
$$

This equation means that given the number $n_{p}$ of photons detected by the HRP sensor, we can predict the number $n_{i}^{\prime}$ of photons that the $\mathrm{LRM}_{i}$ sensor should detect. In terms of our image merging problem, (16) supplies the number of HRP photons, which contain the spatial detail, to be injected into the LRM image.

In this subsection, only sensor spectral sensitivity has been taken into account. In the following subsection, physical properties of the observed object are considered.

\section{B. Physical Emission From Observed Object}

Physical properties of the object define observed radiances and spectral signatures. In the previous subsection, only sensor SRFs were taken into account, which would lead to the wrong conclusion that always the same fraction of HRP photons are added to the $\mathrm{LRM}_{i}$ sensor, independent of the spectral signature of the observed object. For example, if the observed object is a forest, we know that we are mainly recording green and near-infrared (NIR) photons; hence, we have to add more HRP photons to the green and NIR bands of the multispectral sensor than to the other bands. In fact, this is what all image fusion methods intend to accomplish: preserve the spectral signature of the observed objects as much as possible. Since this is not performed by (16), obtained when only considering the sensor contribution, the information from the physical object has to be introduced as well.

Since the contribution to the HRP sensor from every $\mathrm{LRM}_{i}$ sensor is defined by (15), these values can be used to preserve the spectral signature of the observed object when adding HRP photons from the HRP band to every $\mathrm{LRM}_{i}$ band. Therefore, we define

$$
s_{i}=\frac{\rho_{p, i}}{\bar{\rho}_{p}}
$$

as the spectral signature factor of the $\mathrm{LRM}_{i}$ band. The term $\rho_{p, i}$ is the number of photons per frequency unit, which resembles a photon density. It is defined as

$$
\rho_{p, i}=\frac{n_{p, i}}{P\left(m_{i} \cap p_{m}\right)}
$$

which is the number of photons below both the multispectral $\varphi_{i}(\nu)$ and the panchromatic $\phi(\nu)$ functions, divided by the common area below these curves. The term $\bar{\rho}_{p}$ is the mean value for all $\mathrm{LRM}_{i}$ bands, i.e., $\bar{\rho}_{p}=(1 / n) \sum_{i=1}^{n} \rho_{p, i}, n$ being the number of LRM bands. Equation (17) is a way to measure what $\mathrm{LRM}_{i}$ band contains more photons. It gives an idea about the spectral distribution of photons coming from the physical object observed, i.e., the spectral signature.

We can also calculate the $\alpha_{p}$ factor from the data as

$$
\alpha_{p}=\frac{\sum_{\forall i} n_{p, i}}{n_{p, r}}
$$

i.e., as the ratio beetwen the sum of the HRP photons coming from $\mathrm{LRM}_{i}$ bands and the $n_{p, r}$ number of observed photons on the residual plane of the wavelet decomposition of the HRP band. In the former comparison, we use the residual wavelet plane $c_{N}$ instead of the HRP image itself, because the energy of $c_{N}$ is assumed to be equivalent to the sum of the $\mathrm{LRM}_{i}$ bands, i.e., $c_{N}=\sum_{\forall i} m_{i}$. This hypothesis is common to most waveletbased image fusion methods. This estimation of $\alpha_{p}$ factor is better than that in (13), because here we obtain its value from real data instead of from a statistical estimator. Using such information from real data we directly compensate for photons coming from frequency ranges of the HRP SRF that are not covered by any $\mathrm{LRM}_{i} \mathrm{SRF}$.

\section{WiSpeR Algorithm}

Knowing contributions from both the sensor spectral response and physical properties of the object, we combine these effects to obtain a final expression for the merged image. The simplified final expression to the contribution from the HRP band to each of the $\mathrm{LRM}_{i}$ bands is

$$
n_{i}^{f}=s_{i} \cdot n_{i}^{\prime}
$$

noting that in the $n_{i}^{\prime}$ number, the $n_{p}$ HRP photons are substituted by the $\omega$ wavelet coefficient obtained from the HRP band.

Using (16) to include the sensor SRF, and (17) to preserve the spectral signature, we obtain

$$
n_{i}^{f}=\frac{\rho_{p, i}}{\bar{\rho}_{p}} \cdot \frac{\alpha_{p} \cdot P\left(m_{i} \mid p_{m}\right)}{P\left(p_{m} \mid m_{i}\right)} \cdot \omega_{k} .
$$

However, (19) and, by extension, (21) are accurate only when all the $\mathrm{LRM}_{i}$ SRFs do not overlap. In practical situations, this is almost never the case. Hence, some corrections need to be introduced. This effect has to be taken into account both at the time of comparing the number of $\mathrm{LRM}_{i}$ and HRP photons, and when adding the $\omega$ HRP detail photons to the $\mathrm{LRM}_{i}$ bands. 
For example, take the overlap between the $\mathrm{LRM}_{1}$ and $\mathrm{LRM}_{2}$ SRFs, as seen in Fig. 2. In this situation, (19) does not hold, since $n_{p, i}$ LRM photons from the intersection area would be counted twice when trying to compare to the corresponding $n_{p, r}$ HRP photons. In order to compensate for this effect, photons from the intersection area are multiplied by a 0.5 factor. We can calculate the overlapping areas between $\mathrm{LRM}_{i}$ SRFs and introduce these equipartition corrections into (15) and in the $\omega_{k}$ number of HRP photons coming from the $k$ th wavelet plane of the HRP band to be added to the $\mathrm{LRM}_{i}$ band, as

$$
n_{p, i}=P\left(p_{m} \mid m_{i}\right) \cdot n_{i} \cdot\left(1-\frac{\beta_{i}}{2}\right)
$$

finally obtaining

$$
\omega_{i, k}^{f} \equiv n_{i}^{f}=\frac{\rho_{p, i}}{\bar{\rho}_{p}} \cdot \frac{\alpha_{p} \cdot P\left(m_{i} \mid p_{m}\right)}{P\left(p_{m} \mid m_{i}\right)} \cdot\left(1-\frac{\beta_{i}}{2}\right) \cdot \omega_{k}
$$

$\beta_{i}$ being the fraction of the area of the $\mathrm{LRM}_{i} \mathrm{SRF}$ shared with its adjacent $\mathrm{LRM}_{j} \mathrm{SRF}$, and $\omega_{i, k}^{f} \equiv n_{i}^{f}$ the detail from the $\omega_{k}$ wavelet plane of the HRP band to be injected into the $\mathrm{LRM}_{i}$ band.

The final complete expression for the merged $\mathrm{LRM}_{i}$ band is

$$
\begin{aligned}
\operatorname{LRM}_{i}^{f} & =\mathrm{LRM}_{i}+\sum_{k=1}^{n_{\omega}} \omega_{i, k}^{f} \\
& =\mathrm{LRM}_{i}+\sum_{k=1}^{n_{\omega}} \frac{\rho_{p, i}}{\bar{\rho}_{p}} \cdot \frac{\alpha_{p} \cdot P\left(m_{i} \mid p_{m}\right)}{P\left(p_{m} \mid m_{i}\right)} \cdot\left(1-\frac{\beta_{i}}{2}\right) \cdot \omega_{k}
\end{aligned}
$$

$n_{\omega}$ being the number of wavelet planes into which the HRP band is decomposed. To ease the practical implementation of this expression, we want to stress that term $\rho_{p, i}$ is computed using (18), term $\alpha_{p}$ using (19), and terms $P\left(m_{i} \mid p_{m}\right)$ and $P\left(p_{m} \mid m_{i}\right)$ using (9)-(12) and (2).

Equation (24) preserves the spectral signature on the first term, and takes into account the sensor spectral sensitivity on the second and third probabilistic terms. The spectral signature is obtained from the observed $\mathrm{LRM}_{i}$ and HRP data, and the sensor contribution is obtained by integration of the experimental SRFs supplied by the satellite manufacturers. The proposed algorithm is named WiSpeR by the authors, as it stands for window spectral response.

The WiSpeR method described in (24) can be understood as a generalization of different wavelet-based image fusion methods. In particular, we demonstrate in the Appendix that the WiSpeR method generalizes the AWLP method explained above and previously introduced by some of the authors as shown in [34].

\section{EXAMPLES AND ASSESSMENT}

A couple of QuickBird-2 multispectral and panchromatic images, taken in October 2002 and covering both agricultural and urban areas of Sevilla, Spain (Fig. 2) were used as LRM and HRP test images to evaluate the performance of the WiSpeR fusion method. SRFs of Quickbird-2 are shown in Fig. 1.

The quality of the resulting WiSpeR merged image (HRM) was compared with that corresponding to the HRM images obtained applying the following standard wavelet-based methods:

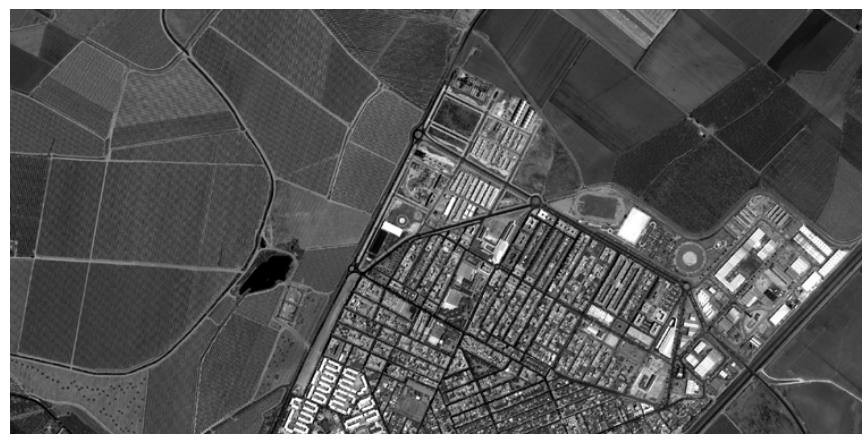

Fig. 2. QuickBird-2 HRP image, at a resolution of $0.7 \mathrm{~m}, 4096$ columns by 2048 rows.

-additive wavelet-based method (AW), similar to that used in [26]-[30] amongst others, injecting the spatial detail of the HRP image into each band of the LRM, using the undecimated à trous algorithm to perform the spatial detail extraction of the HRP image and a histogram matching transformation model to preserve the spectral information of the multispectral original image;

- proportional additive wavelet intensity method (AWLP), based on the AWL method proposed in [34], and previously described in (1).

The spatial resolution of the QuickBird-2 HRP and LRM images are 0.7 and $2.8 \mathrm{~m}$, respectively. The HRM images obtained after the fusion would show a spatial resolution similar to that of the HRP image, in this case $0.7 \mathrm{~m}$. In order to assess the quality of the merged images, they should be compared with the image that the QuickBird-2 sensor would theoretically collect in multispectral mode if it had a spatial resolution of $0.7 \mathrm{~m}$. Since these images do not exist, we worked with spatially degraded images. Thus, before the fusion process, the QuickBird-2 HRP and LRM images were degraded to 2.8 and $11.2 \mathrm{~m}$. The goodness of each image-fusion method is evaluated by comparing the resulting merged images with the QuickBird-2 LRM original image.

This comparison is based both on spectral and spatial characteristics.

\section{A. Spectral Quality Assessment}

A good fusion method has to guarantee the preservation of the spectral information of the multispectral image when increasing its spatial information. To measure the spectral distortion due to the fusion process, each merged image is compared to the original QuickBird-2 multispectral image, using the following quantitative indicators [11].

-Correlation coefficient (CC) between each band of the original and the merged image. It should be as close to 1 as possible.

- Root mean square error (RMSE) between each band of the original and the merged image, in radiance, computed using the following expression:

$$
\mathrm{RMSE}^{2}=\operatorname{bias}^{2}+\mathrm{SDD}^{2}
$$

where the bias is the difference between the mean value of the original and merged bands and SDD the standard deviation of the difference image between each original and merged bands. RMSE should be as close to 0 as possible. 
These parameters only allow evaluating the difference in spectral information between corresponding bands of the merged image and the original image. In order to estimate the global spectral quality of the merged images, we have used the following parameters.

The erreur relative globale adimensionnelle de synthèse index (ERGAS), whose english translation is relative dimensionless global error in fusion [40], given by

$$
\text { ERGAS }=100 \frac{h}{l} \sqrt{\frac{1}{N} \sum_{i=1}^{N}\left(\frac{\operatorname{RMSE}^{2}\left(B_{i}\right)}{M_{i}^{2}}\right)}
$$

where $h$ is the resolution of the HRP image, $l$ the resolution of the LRM image, $N$ the number of spectral bands $\left(B_{i}\right)$ involved in the fusion, and $M_{i}$ the mean radiance of each spectral band. The lower the ERGAS value, the higher the spectral quality of the merged images.

- The average quality index $\left(Q_{\mathrm{avg}}\right)$ based on the universal quality index $(Q)$ proposed by [41] and given by

$$
Q=\frac{4 \sigma_{x y} \cdot \bar{x} \cdot \bar{y}}{\left(\sigma_{x}^{2}+\sigma_{y}^{2}\right)\left[(\bar{x})^{2}+(\bar{y})^{2}\right]}
$$

where $\bar{x}$ and $\bar{y}$ are the mean of each original $(x)$ and fused ( $y$ ) images; $\sigma_{x}^{2}$ and $\sigma_{y}^{2}$ are the variances of $x$ and $y$; and $\sigma_{x y}$ is the covariance between $x$ and $y$.

This $Q$ index models the difference between two images as a combination of three different factors: loss of correlation, luminance distortion and contrast distortion. As image quality is often space dependent [41], the $Q$ index has been calculated using a sliding window approach. In this work, sliding windows with a size of $8 \times 8,16 \times 16,32 \times 32,64 \times 64$, and $128 \times 128$ pixels are used.

Considering that the $Q$ index can only be applied to monochromatic images, the average value $\left(Q_{\text {avg }}\right)$ is used as a global spectral quality index for multispectral images. The higher the $Q_{\text {avg }}$ value the higher the spectral and radiometric quality of the merged images.

The values of these parameters resulting from the comparison of the merged images and the original QuickBird-2 multispectral image are reported in Table I.

In order to quantify the effect of the fusion process on the initial multispectral image (11.2 m per pixel, spatially degraded image), we show in the first column the values of the quantitative indicators obtained when this degraded image was compared with the original multispectral image $(2.8 \mathrm{~m}$ per pixel). This first column reflects the situation before the fusion, while the last column reflects the situation that ideally should be reached after the fusion process.

To ease the comparison of the different fusion methods according to the $Q_{\text {avg }}$ parameter, we have displayed the $Q_{\text {avg }}$ values for different sliding size windows in Fig. 3.

Lower CC and $Q_{\text {avg }}$ or higher RMSE and ERGAS values than those showed in the first column indicate that the analyzed image-fusion algorithm tends to distort the spectral information of the initial multispectral image. It can be observed that all fusion methods based on wavelets allow a high-quality transformation of the multispectral information when increasing its spatial resolution (Fig. 4).
TABLE I

VALUE OF THE DIFFERENT PARAMETERS ANALYZED TO EVALUATE

\begin{tabular}{|c|c|c|c|c|c|c|}
\hline & & $\mathrm{X}_{\text {degraded }}$ & $\mathrm{AW}$ & AWLP & WiSpeR & IDEAL \\
\hline \multirow[t]{4}{*}{$\mathrm{CC}$} & $\mathrm{X} 1$ & 0.9489 & 0.9492 & 0.9543 & 0.9662 & 1 \\
\hline & $\mathrm{X} 2$ & 0.9433 & 0.9554 & 0.9702 & 0.9716 & 1 \\
\hline & $\mathrm{X} 3$ & 0.9409 & 0.9573 & 0.9736 & 0.9726 & 1 \\
\hline & $\mathrm{X} 4$ & 0.8960 & 0.9410 & 0.9403 & 0.9481 & 1 \\
\hline \multirow[t]{4}{*}{ RMSE } & $\mathrm{X} 1$ & 0.4246 & 0.4884 & 0.4207 & 0.3695 & 0 \\
\hline & $\mathrm{X} 2$ & 0.6136 & 0.5906 & 0.4554 & 0.4493 & 0 \\
\hline & $\mathrm{X} 3$ & 0.6079 & 0.5606 & 0.4156 & 0.4186 & 0 \\
\hline & $\mathrm{X} 4$ & 1.1545 & 0.8899 & 0.8846 & 0.8683 & 0 \\
\hline ERGAS & & 2.942 & 2.769 & 2.227 & 2.104 & 0 \\
\hline$Q_{\text {avg }} 8 \times 8$ & & 0.440 & 0.600 & 0.630 & 0.638 & 1 \\
\hline Qavg $16 \times 16$ & & 0.577 & 0.684 & 0.712 & 0.726 & 1 \\
\hline Qavg $32 \times 32$ & & 0.693 & 0.768 & 0.793 & 0.810 & 1 \\
\hline Qavg $64 \times 64$ & & 0.786 & 0.843 & 0.863 & 0.880 & 1 \\
\hline Qavg $128 \times 128$ & & 0.844 & 0.888 & 0.904 & 0.920 & 1 \\
\hline
\end{tabular}
THE SPECTRAL Quality OF THE MERGEd IMAGES

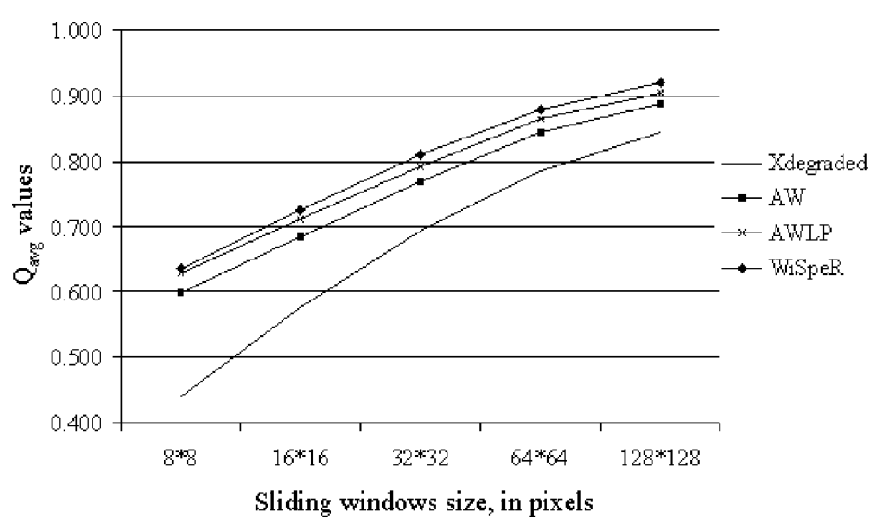

Fig. 3. Graphical representation of the $Q_{\text {avg }}$ values of the QuickBird-2 merged images for different sliding size windows.

The WiSpeR method provides an HRM image of higher spectral quality than the AW and AWLP methods, because the transformation model is not based on global image information, as is the case of the histogram matching process, but based on pixel information. Taking into account both pixel spectral signature and the SRFs of QuickBird-2 sensors, a selective incorporation of spatial detail of the HRP image into each band of the LRM image is performed. This procedure allows obtaining an HRM image closer to the image that the multispectral sensor would capture if it worked at a spatial resolution equal to that of the panchromatic one.

It is important to point out that the wavelet coefficients injected into each LRM band, when the WiSpeR method is used, have been extracted from the original HRP-radiance image. However, when the AW and AWLP methods are used, the wavelet coefficients have been extracted from a modified HRP-radiance image (i.e., after a histogram matching process.) If these wavelet coefficients were extracted from the original HRP-radiance image, the quality of the resulting merged images would decrease significantly.

For illustrative purposes, we show a detail of another Quickbird image in Fig. 5. Image fusion methods, even wavelet-based ones 


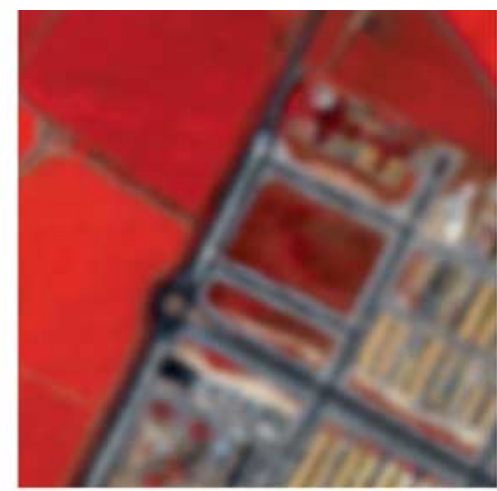

(a)

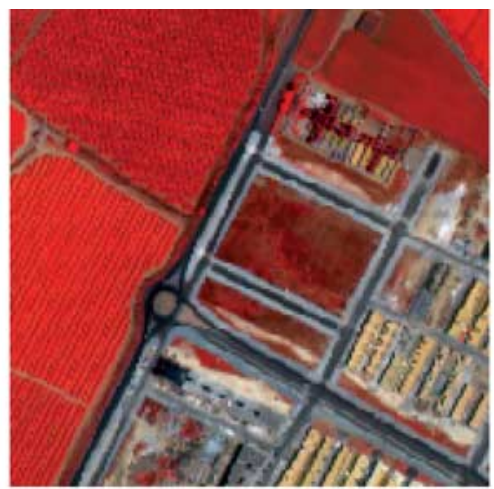

(b)

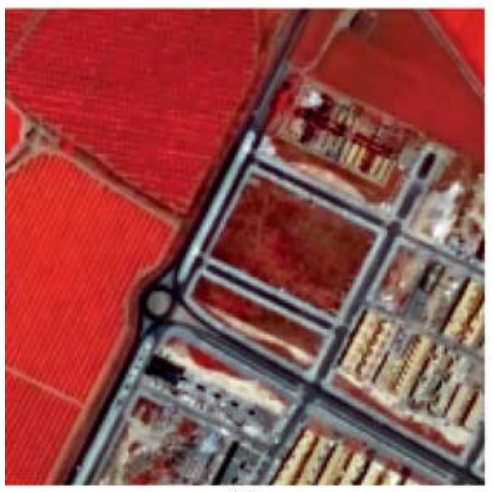

(c)

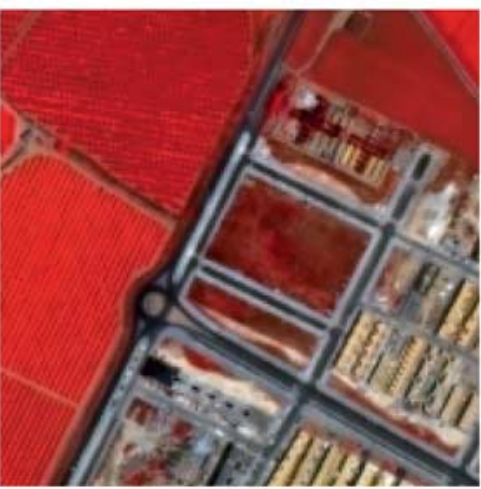

(d)

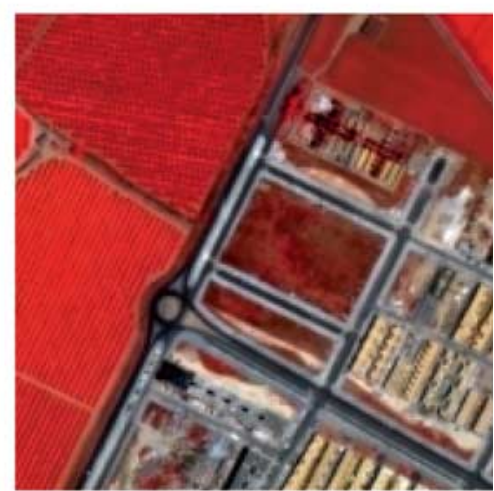

(e)

Fig. 4. Color composition (160 rows $\times 160$ columns) corresponding to (a) multispectral initial, spatially degraded, image, (b) X1 multispectral original image, (c) multispectral AW merged image, (d) multispectral AWLP merged image, and (e) multispectral WiSpeR image.

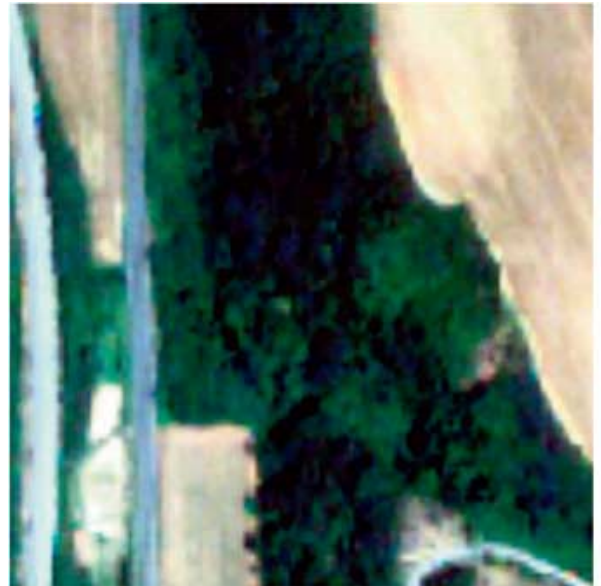

(a)

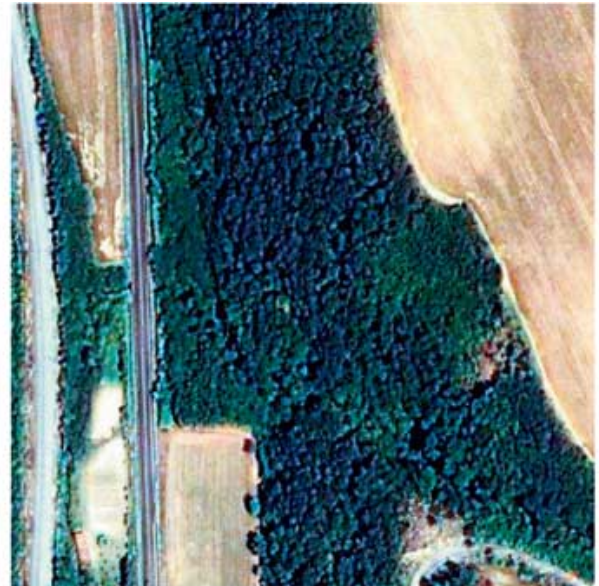

(b)

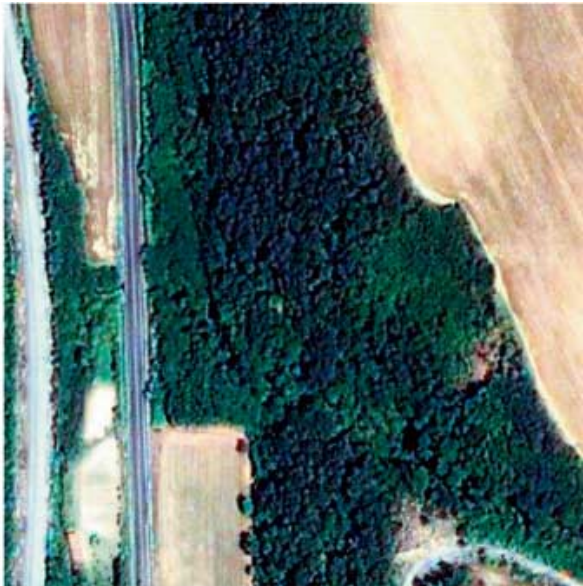

(c)

Fig. 5. Color composition (400 rows $\times 400$ columns) corresponding to (a) multispectral initial, spatially degraded, image, (b) multispectral AWLP merged image, and (c) multispectral WiSpeR merged image. Images courtesy of Eurimage.

like the AWLP method, show some problems when the radiance values of the observed LRM bands are very different and some LRM SRFs overlap. This problem can be observed in forest areas, where the visual bands show low radiance values, but the NIR band show high radiance values. The AWLP method produces bluish pixels on some forest pixels because the spectral response of the sensor is not taken into account by this kind of image fusion methods. Blue and green LRM SRFs overlap. Hence, when injecting HRP details into these bands using the AWLP method, some photons are injected twice into these two bands, creating a green and, overall, blue photon surplus into these bands, i.e., the bluish pixels in the final merged image in Fig. 5(b). When the overlapping of LRM SRFs is taken into account, the correct number of photons is introduced into every LRM band, avoiding the excess of blue and green photons and producing better results. This problem is solved by the WiSpeR method, which correctly distributes HRP photons between the LRM bands producing green, instead of bluish, pixels in forest areas.

\section{B. Spatial Quality Assessment}

A good fusion method must allow the injection, into each band of the multispectral image, of that spatial detail the multi- 
TABLE II

SCC VALUES RESUlting From the COMPARISON BETWEEN THE SPATIAL DeTAIL OF THE ORIGINAL QUiCKBIRD-2 MulTiSPECTRAL IMAGE AND THE SPATIAL DETAIL OF THE DIFFERENT MERGED IMAGES

\begin{tabular}{ccccccc}
\hline \hline & & X $_{\text {degraded }}$ & AW & AWLP & WiSpeR & IDEAL \\
\hline SCC & X1 & 0.3913 & 0.5325 & 0.5453 & 0.5854 & 1 \\
& X2 & 0.4004 & 0.6036 & 0.6202 & 0.6426 & 1 \\
& X3 & 0.3952 & 0.5640 & 0.5944 & 0.6121 & 1 \\
& X4 & 0.3716 & 0.6342 & 0.6423 & 0.6681 & 1 \\
\hline \hline
\end{tabular}

spectral sensor would observe if it worked at a spatial resolution similar to that of the panchromatic sensor.

As previously mentioned, the fusion methods proposed and analyzed in this work have been carried out on spatially degraded QuickBird-2 images. This way, the original QuickBird-2 multispectral image is available to evaluate the amount of spatial detail of the panchromatic image that ideally should be injected, during the fusion process, into each band of the multispectral image.

In order to estimate the spatial quality of the merged images, the spatial information present in each band of these images will be compared with the spatial information present in each band of the original QuickBird-2 multispectral image. To do this, we follow the procedure proposed by Zhou [29]. First, the spatial detail information present in the two images to be compared is extracted using a Laplacian filter. Second, the correlation between these two filtered images is calculated. A high spatial correlation coefficient (SCC) indicates that many of the spatial detail information of one of the images is present in the other one.

In this work, the Laplacian filter used to extract the spatial detail of the different images is

$$
\left|\begin{array}{ccc}
-1 & -1 & -1 \\
-1 & 8 & -1 \\
-1 & -1 & -1
\end{array}\right|
$$

In Table II, we show the SCC values obtained when the original QuickBird-2 multispectral image is compared with the resulting merged images. The first column shows the spatial correlation between the original multispectral image and the initial image (degraded image). This column reflects the situation before the fusion, while the last one reflects what would be the ideal ending situation after the fusion process.

Generally speaking, in all the images captured by a remote sensing sensor it is possible to find physical features that are noticeable or separable from other features at a specific range of wavelengths but not at others, independent of the spatial resolution of the sensor that observes these features. This fact should be taken into account when images captured by different sensors, with different detection systems, have to be fused. The WiSpeR method proposed in this work takes into account not only each pixel signature in the multispectral image to be fused, but also the SRFs of both multispectral and panchromatic sensors, to determine in each band, the amount of spatial detail of the panchromatic image to be injected.

The SCC value of each band of the merged image obtained applying the WiSpeR method is closer to the "ideal" situation than those corresponding to the AW and AWLP merged images when its spatial detail is compared with that of the original QuickBird-2 multispectral one.

\section{CONCLUSION}

Image fusion methods try to obtain the image a sensor would obtain if it had the same spectral response of the original LRM bands but the spatial resolution of the HRP sensor. Since the latter sensor has different spectral information than the LRM sensor, their relative spectral responses have to be taken into account to avoid merging information from unconnected physical electromagnetic spectrum regions.

In previous image fusion methods, physical spectral responses of sensors have not been taken into account. The WiSpeR method developed here incorporates this information into the image fusion process, to determine the amount of spatial detail of the HRP image that has to be injected into each LRM band. Although the detail traction process is applied directly to the original HRP-radiance images, the resulting merged images present better quality than those obtained applying standard wavelet-based methods, because in the latter the HRP-radiance image is transformed, usually through a histogram matching, before the detail extraction process, whereas the WiSpeR method works with the original raw radiance information.

The proposed WiSpeR method is a generalization of other wavelet-based image fusion methods, being equivalent in some particular situations.

As shown in Tables I and II, the WiSpeR method performs better than other wavelet-based fusion methods, both regarding spectral and spatial quality.

\section{APPENDIX}

Let all LRM SRF functions be independent and their union equivalent to the HRP SRF, i.e.,

$$
\begin{aligned}
\bigcap_{i} \varphi_{i}(\nu) & =\varnothing \Rightarrow \beta_{i}=0 \\
\varphi_{i}(\nu) \cap \phi(\nu) & =\varphi_{i}(\nu) \forall i \\
\sum_{\forall i} \varphi_{i}(\nu) & =\phi(\nu) \Rightarrow \alpha_{p}=1 .
\end{aligned}
$$

This implies that from (5), we obtain $P\left(m_{i} \cap p\right)=$ $\int \min \left(\varphi_{i}, \phi\right) d \nu=\int \varphi_{i} d \nu=P\left(m_{i}\right)$. We make the assumption that all the $\mathrm{LRM}_{i}$ SRFs have equal probability and that $P(p)=1$. This implies $P\left(m_{i}\right)=P\left(m_{j}\right)=1 / n, \forall i \neq j, n$ being the number of LRM bands.

From (6) we obtain $P\left(p \mid m_{i}\right)=1$, and from (4) we obtain $P\left(m_{i} \mid p\right)=P\left(m_{i}\right) / P(p)=1 / n$. This means that from (15) we obtain $n_{p, i}=P\left(p_{m} \mid m_{i}\right) \cdot n_{i}=n_{i}$; hence from (18) we obtain

$$
\begin{aligned}
\rho_{p, i} & =\frac{n_{i}}{\frac{1}{n}}=n \cdot n_{i} \\
\bar{\rho}_{p} & =\frac{1}{n} \sum_{\forall i} n \cdot n_{i}=\sum_{\forall i} n_{i} \\
s_{i} & =\frac{\rho_{p, i}}{\bar{\rho}_{p}}=\frac{n \cdot n_{i}}{\sum_{\forall i} n_{i}} .
\end{aligned}
$$


Developing (23)

$$
\begin{aligned}
n_{i}^{f} & =\frac{\rho_{p, i}}{\bar{\rho}_{p}} \cdot \frac{\alpha_{p} \cdot P\left(m_{i} \mid p_{m}\right)}{P\left(p_{m} \mid m_{i}\right)} \cdot\left(1-\frac{\beta_{i}}{2}\right) \cdot \omega \\
& =\frac{n \cdot n_{i}}{\sum_{\forall i} n_{i}} \cdot \frac{1 \cdot \frac{1}{n}}{1} \cdot\left(1-\frac{0}{2}\right) \cdot \omega
\end{aligned}
$$

we finally obtain

$$
n_{i}^{f}=\frac{n_{i}}{\sum_{\forall i} n_{i}} \cdot \omega
$$

which is the same expression as (1).

That proves that the AWLP method is a particular case of the WiSpeR method, and they are equivalent when the LRM SRF functions satisfy the following conditions.

- There is no overlap between them.

- Are equally probable.

- Their union is equivalent to the HRP SRF.

\section{ACKNOWLEDGMENT}

The authors thank one of the anonymous reviewers for extremely detailed, accurate, and useful comments about the paper. The authors thank J. L. Saleta for his useful comments about this paper, and the authors thank Eurimage for the supply of the panchromatic and multispectral images used for the example in Fig. 5.

\section{REFERENCES}

[1] R. Haydn, G. W. Dalke, J. Henkel, and J. E. Bare, "Application of HIS color transform to the processing of multisensor data and image enhancement," in Proc. Int. Symp. Remote Sensing of Arid and Semi-Arid Lands, Cairo, Egypt, 1982, pp. 599-616.

[2] J. W. Carper, T. M. Lillesand, and R. W. Kiefer, "The use of intensity-hue-saturation transformations for merging SPOT panchromatic and multispectral image data," Photogramm. Eng. Remote Sens., vol. 56, pp. 459-467, 1990 .

[3] C. Pohl and J. L. van Genderen, "Multisensor image fusion in remote sensing: Concepts, methods and applications," Int. J. Remote Sens., vol. 19, pp. 823-854, 1998

[4] P. S. Chavez, Jr, S. C. Sides, and J. A. Anderson, "Comparison of three different methods to merge multiresolution and multispectral data: Landsat TM and SPOT panchromatic," Photogramm. Eng. Remote Sens., vol. 57, no. 3, pp. 295-303, 1991.

[5] M. Ehlers, "Multisensor image fusion techniques in remote sensing," ISPRS J. Photogramm. Remote Sens., vol. 51, pp. 311-316, 1991.

[6] V. K. Shettigara, "A generalized component substitition technique for spatial enhacement of multispectral images using a higher resolution data set," Photogramm. Eng. Remote Sens., vol. 58, pp. 561-567, 1992.

[7] G. Cliche and F. Bonn, "Integration of SPOT panchromatic channel into multispectral mode for image sharpness enhancement," Photogramm. Eng. Remote Sens., vol. 51, no. 3, pp. 811-816, 1985.

[8] SPOT Image, "SPOT Users Handbook", vol. 1-3, Centre National d'Etude Spatiale (CNES) and SPOT Image, Toulouse, France, 1988.

[9] A. R. Gillespie, A. B. Kahle, and R. E. Walker, "Color enhancement of highly correlated images II. Channel ratio and chromaticity transformation technique," Remote Sens. Environ., vol. 22, no. 3, pp. 343-365, 1987.

[10] C. Pohl, "Geometric aspects of multisensor image fusion for topographic map updating in the humid tropics," Int. Inst. Aerospace Surv. Earth Sci., Enschede, The Netherlands, ITC Pub. 39, 1996.

[11] L. Wald, T. Ranchin, and M. Mangolini, "Fusion of satellite images of different spatial resolutions: Assessing the quality of resulting images," Photogramm. Eng. Remote Sens., vol. 63, no. 6, pp. 691-699, 1997.
[12] M. González de Audícana, R. García, and J. Herrero-Isern, "Fusion of multispectral and panchromatic images using wavelet transform. Evaluation of crop classification accuracy," in Proc. 22nd EARSeL Аnпu. Symp. "Geoinformation for European-Wide Integration", vol. 1, T. Benes, Ed., Prague, Czech Republic, Jun. 4-6, 2002, pp. 265-272.

[13] T. Ranchin and L. Wald, "Merging SPOT-P and KVR-1000 for updating urban maps," in Proc. 26th Int. Symp. Remote Sensing of Environment and the 18th Annu. Symp. Can. Remote Sensing Soc., Vancouver, BC, Canada, Mar. 25-29, 1996, pp. 262-265.

[14] P. Terretaz, "Comparison of different methods to merge SPOT P and XS data: Evaluation in an urban area," in Proc. 17th Symp. EARSeL, Future Trends in Remote Sensing, P. Gudmansen, Ed., Lyngby, Denmark, Jun. 20, 1997, pp. 435-445.

[15] I. Couloigner, T. Ranchin, and L. Wald, "Benefit of data fusion to urban roads mapping," in Proc. 2nd Conf. "Fusion of Earth Data: Merging Point Measurements, Raster Maps and Remotely Sensed Images", T. Ranchin and L. Wald, Eds., Sophia Antipolis, France, Jan. 28-30, 1998, pp. 183-190.

[16] V. S. Raptis, R. A. Vaughan, T. Ranchin, and L. Wald, "Assessment of different data fusion methods for the classification of an urban environment," in Proc. 2nd Conf. "Fusion of Earth Data: Merging Point Measurements, Raster Maps and Remotely Sensed Images", T. Ranchin and L. Wald, Eds., Sophia Antipolis, France, Jan. 28-30, 1998, pp. 167-182.

[17] I. Couloigner, T. Ranchin, V. P. Valtonen, and L. Wald, "Benefit of the future SPOT 5 and of data fusion to urban mapping," Int. J. Remote Sens., vol. 19, no. 8, pp. 1519-1532, 1998.

[18] Y. Cornet, S. de Béthune, M. Binard, F. Muller, G. Legros, and I. Nadasdi, "RS data fusion by local mean and variance matching algorithms: Their respective efficiency in a complex urban context," in Proc. IEEE/ISPRS Joint Workshop on Remote Sensing and Data Fusion Over Urban Areas, Rome, Italy, Nov. 8-9, 2001, pp. 105-111.

[19] A. Fanelli, A. Leo, and M. Ferri, "Remote sensing images data fusion: A wavelet transform approach for urban analysis," in Proc. IEEE/ISPRS Joint Workshop on Remote Sensing and Data Fusion Over Urban Areas, Rome, Italy, Nov. 8-9, 2001, pp. 112-116.

[20] D. D. Kishore, R. K. Gopal, and A. Prakash, "Improvement of effective spatial resolution of thermal infrared data for urban landuse classification," in Proc. IEEE/ISPRS Joint Workshop on Remote Sensing and Data Fusion Over Urban Areas, Rome, Italy, Nov. 8-9, 2001, pp. 332-336.

[21] D. Vaiopoulos, K. Nikolakopoulos, and G. Skianis, "A comparative study of resolution merge techniques and their efficiency in processing image of urban areas," in Proc. IEEE/ISPRS JointWorkshop on Remote Sensing and Data Fusion Over Urban Areas, Rome, Italy, Nov. 8-9, 2001, pp. 270-274.

[22] L. Wald and T. Ranchin, "Data fusion for a better knowledge of urban areas," in Proc. IEEE/ISPRS Joint Workshop on Remote Sensing and Data Fusion Over Urban Areas, Rome, Italy, Nov. 8-9, 2001, pp. $127-132$.

[23] I. Daubechies, Ten Lectures on Wavelets. Philadelphia, PA: SIAM, 1992, CBMS-NSR Regional Conference Series in Applied Mathematics.

[24] C. H. Chui, An Introduction to Wavelets. Boston, MA: Academic, 1992.

[25] S. Mallat, A Wavelet Tour of Signal Processing, 2nd ed. Orlando, FL: Academic, 1999.

[26] T. Ranchin and L. Wald, "Fusion of high spatial and spectral resolution images: The ARSIS concept and its implementation," Photogramm. Eng. Remote Sens., vol. 66, pp. 49-61, Jan. 2000.

[27] B. Garguet-Duport, J. Girel, J. M. Chasseny, and G. Pautou, "The use of multiresolution analysis and wavelet transform for merging SPOT panchromatic and multispectral image data," Photogramm. Eng. Remote Sens., vol. 62, pp. 1057-1066, Sep. 1996.

[28] D. A. Yocky, "Image merging and data fusion by means of the discrete two-dimensional wavelet transform," J. Opt. Soc. Amer. A., vol. 12, pp. 1834-1841, Sep. 1995.

[29] J. Zhou, D. L. Civco, and J. A. Silander, "A wavelet transform method to merge Landsat TM and SPOT panchromatic data," Int. J. Remote Sens., vol. 19 , no. 4 , pp. $743-757,1998$.

[30] M. Mangolini, T. Ranchin, and L. Wald, "Fusion d'images SPOT multispectrale (XS) et panchromatique (P), et d'images radar," in Actes du Colloque SPOT-ERS: De l'Optique au Radar. Les Applications de SPOT et ERS, 1993, pp. 199-209.

[31] B. Aiazzi, L. Alparone, S. Baronti, and A. Garzelli, "Context-driven fusion of high spatial and spectral resolution images based on oversampled multiresolution analysis," IEEE Trans. Geosci. Remote Sens., vol. 40, no. 10, pp. 2300-2312, Oct. 2002. 
[32] M. González-Audícana, J. L. Saleta, O. G. Catalán, and R. García, "Fusion of multispectral and panchromatic images using improved IHS and PCA mergers based on wavelet decomposition," IEEE Trans. Geosci. Remote Sens., vol. 42, no. 6, pp. 1291-1299, Jun. 2004.

[33] M. González-Audícana, X. Otazu, O. Fors, and A. Seco, "Comparison between the Mallat's and the 'à trous' discrete wavelet transform based algorithms for the fusion of multispectral and panchromatic images," Int. J. Remote Sens., vol. 26, no. 3, pp. 597-616, Feb. 2005.

[34] J. Nuñez, X. Otazu, O. Fors, A. Prades, V. Pala, and R. Arbiol, "Multiresolution-based image fusion with additive wavelet decomposition," IEEE Trans. Geosci. Remote Sens., vol. 37, no. 3, pp. 1204-1211, May 1999.

[35] Y. Chibani and A. Houacine, "The joint use of IHS transform and redundant wavelet decomposition for fusing multispectral and panchromatic images," Int. J. Remote Sens., vol. 23, pp. 3821-3833, Sep. 2002.

[36] M. González-Audícana, X. Otazu, O. Fors, R. García, and J. Núñez, "Fusion of different spatial and spectral resolution images: Development, application and comparison of new methods based on wavelets," in Proc. Int. Symp. Recent Advances in Quantitative Remote Sensing, Sep. 2002, pp. 228-237.

[37] M. Mangolini, "Apport de la fusion d'images satellitaires multicapteurs au niveau pixel en télédétection et photo-interprétation,” Eng. Doctoral, Univ. Nice-Sophia Antipolis, France, 1994.

[38] T. Ranchin, B. Aiazzi, L. Alparone, S. Baronti, and L. Wald, "Image fusion-The ARSIS concept and some successful implementation schemes," ISPRS J. Photogramm. Remote Sens., vol. 58, pp. 4-18, 2003.

[39] M. Holschneider, R. Kronland-Martinet, J. Morlet, and P. Tchamitchian, "Chapter a real-time algorithm for signal analysis with the help of the wavelet transform," in Wavelets, Time-Frequency Methods and Phase Space. Berlin, Germany: Springer-Verlag, 1989, pp. 289-297.

[40] L. Wald, "Quality of high resolution synthesized images: Is there a simple criterion?," in Proc. Int. Conf. Fusion of Earth Data, vol. 1, Nice, France, Jan. 2000, pp. 99-105.

[41] Z. Wang and A. C. Bovik, "A universal image quality index," IEEE Signal Process. Lett., vol. 9, no. 3, pp. 81-84, Mar. 2002.

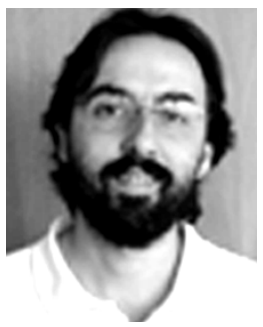

Xavier Otazu received the B.A. degree in physics from the University of Barcelona, Barcelona, Spain, the M.S. degree in remote sensing from the Institut d'Estudis Espacials de Catalunya, Barcelona, and $\mathrm{Ph} . \mathrm{D}$. degree in physics from the University of Barcelona in 1994, 1999, and 2001, respectively.

Since 1994, he has been working on applications of wavelets to astronomical and remote sensing image and data processing techniques such as image deconvolution, image fusion, period analysis, and data clustering. He has also been working in the $\mathrm{R}+\mathrm{D}$ remote sensing group at the Institut Cartogràfic de Catalunya on SAR image processing, image fusion, and image classification. He is currently a Ramón y Cajal Researcher at the Computer Vision Center and a Lecturer in the Computer Science Department, Universitat Autonoma de Barcelona, working in color and texture image analysis, especially in the study of the human color visual system and the mathematical description of its perceptual processes using wavelets.

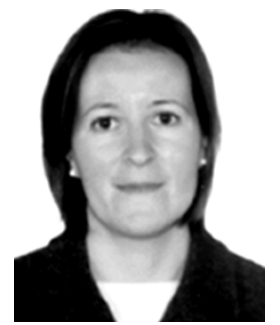

María González-Audícana received the B.S. and $\mathrm{Ph} . \mathrm{D}$. degrees in agricultural engineering, with a thesis based on image-fusion, from the Public University of Navarre, Pamplona, Spain, in 1996 and 2001, respectively.

Since 1997, she has been an Assistant Professor with the Department of Projects and Rural Engineering, Public University of Navarre, where she is currently involved in research activities on multisensor data fusion, multispectral image segmentation, and image classification for agricultural management purposes.

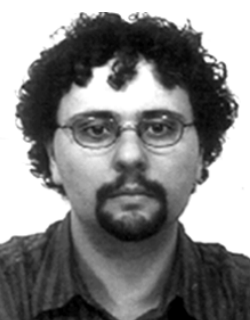

Octavi Fors received the B.A. degree in physics from the University of Barcelona, Barcelona, Spain, and the M.S. degree in remote sensing from the Institut d'Estudis Espacials de Catalunya, Barcelona, in 1996 and 1999, respectively. He is currently pursuing the Ph.D. degree in physics at the University of Barcelona.

Since 1996, he has been working on image processing techniques both in the astronomy and remote sensing fields. In particular, he has developed a special interest in image deconvolution, image fusion, and multiresolution-based disparity analysis.

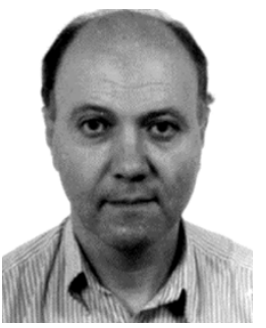

Jorge Núñez received the B.A. and Ph.D. degrees in physics from the University of Barcelona, Barcelona, Spain, in 1975 and 1981, respectively.

Since 1984, he has been a permanent Professor in the Department of Astronomy, University of Barcelona. In 1998, he was elected a member of the Academy of Sciences and Arts of Barcelona. $\mathrm{He}$ is currently conducting research on digital image processing including image reconstruction and restoration using Bayesian techniques and data fusion with applications in astronomy, medicine, and remote sensing. 